\title{
Inherent and Local Magnetic Field Structures in Jets from Active Galactic Nuclei
}

\author{
Denise C. Gabuzda
}

Citation: Gabuzda, D.C. Inherent and Local Magnetic Field Structures in Jets from Active Galactic Nuclei. Galaxies 2021, 9, 58. https://doi.org/ 10.3390 /galaxies 9030058

Academic Editors: Margo Aller, Jose L. Gómez and Eric Perlman

Received: 20 July 2021

Accepted: 19 August 2021

Published: 25 August 2021

Publisher's Note: MDPI stays neutral with regard to jurisdictional claims in published maps and institutional affiliations.

Copyright: (C) 2021 by the author. Licensee MDPI, Basel, Switzerland. This article is an open access article distributed under the terms and conditions of the Creative Commons Attribution (CC BY) license (https:/ / creativecommons.org/licenses/by/ $4.0 /)$.
Physics Department, University College Cork, T12 K8AF Cork, Ireland; d.gabuzda@ucc.ie; Tel.: +353-21-490-2003
Abstract: In theoretical models for the electromagnetic launching of astrophysical jets, a helical magnetic (B)-field component is generated through the winding up of an initial longitudinal field component by the rotation of the cental black hole and accretion disk. This helical field component travels outward with the jet plasma. There is now abundant evidence that the jets of active galactic nuclei carry helical $\mathbf{B}$ fields, and the presence of such fields has been invoked to explain a wide range of phenomena observed in these jets. However, distinguishing between features associated with this inherent jet $\mathbf{B}$ field and with $\mathbf{B}$ fields generated by local phenomena such as shocks and shear can be challenging. There is now evidence that the field that is accreted is dipolar like, giving rise to a current distribution with inward currents along both jet axes and outward currents in a more extended region around the jets. Striking limb brightening has been observed for several relatively nearby active galactic nuclei; it is argued that this must be due to some intrinsic property of the jet, which is independent of the viewing angle, such as its helical $\mathbf{B}$ field, or mass loading and/or particle acceleration at the jet edges. Circular-polarization observations may make it possible to reconstruct the full three-dimensional B field of jets carrying a helical B-field component, and to correctly infer the direction of rotation of the central black hole and its accretion disk.

Keywords: active galactic nuclei; relativistic jets; magnetic fields; radio interferometry

\section{Introduction}

\subsection{Background}

While the radiation of most galaxies is dominated by thermal emission from stars and gas, the luminosities of a small fraction of "active" galaxies are vastly dominated by non-thermal radiation in compact regions in their nuclei. The origin of the tremendous energy radiated is the conversion of gravitational energy to radiation via accretion onto a supermassive black hole ( $\sim 10^{8}-10^{9}$ solar masses) at the galactic centre.

For reasons that are not clear, in a significant minority of active galactic nuclei (AGNs), the accretion process leads to the ejection of "jets" of relativistic plasma from a region near the central black hole. It is usually assumed that these jets are ejected along the black hole's rotational axis, although it is not possible to verify this through direct imaging. The plasma in the jet carries magnetic (B) fields, leading to the emission of radio synchrotron radiation by relativistic electrons present in the jet plasma. Approximately 10-15\% or so of all AGNs, said to be "radio-loud", display substantial amounts of synchrotron emission at centimetre wavelengths (ratio of the $5 \mathrm{GHz}$ flux to the optical B-band flux $\geq 10 ;[1]$ ), and are taken to host radio jets, whether or not these jets are detected directly in images.

The only way to obtain direct information about the orientation and degree of order of the synchrotron B field is through linear-polarization observations. The fact that the radio emission is synchrotron radiation is confirmed by its appreciable degree of linear polarization, which can approach $40-50 \%$ in individual jet features (e.g., [2]). This is fairly close to the theoretical maximum degree of linear polarization for synchrotron radiation from a region with perfectly ordered $\mathbf{B}$ fields and a random pitch-angle distribution for the relativistic electrons [3], $\simeq 75 \%$, indicating that the jet $\mathbf{B}$ fields can sometimes be very well 
ordered. More typically, the degrees of linear polariation in the jets are of order $5-15 \%$, also suggesting the presence of an appreciable tangled field component. Very low observed degrees of polarization indicate either a highly disordered field or the presence of effects leading to depolarization of initially polarized emission.

The direction of the observed plane of linear polarization, or electric vector position angle (EVPA) is orthogonal to the synchrotron $\mathbf{B}$ field in optically thin emission regions, and parallel to the synchrotron B field in sufficiently optically thick regions. It has often been assumed in the past that this transition from optically thin to optically thick occurs near an optical depth $\tau \simeq 1$. However, the associated $90^{\circ}$ rotation in the observed EVPA actually occurs at an optical depth $\tau \simeq 6-7$ [4], far upstream of the observed "core" - the bright feature at one end of jet, often taken to be the location along the jet where the optical depth is near unity [5]. Therefore, virtually all the polarized emission we observe in radio images of AGN jets arises in optically thin regions, including polarization from the core region.

The scales closest to the "central engine" and jet-launching region on which we can obtain direct information are those probed by very long baseline interferometry (VLBI). The jets of AGNs are present on the smallest scales on which they have been targeted using VLBI; a recent example is the report of $\lambda 1.3 \mathrm{~mm}$ VLBI observations of the innermost jets of Centaurus A [6], which sampled scales of order $200 R_{g}$, where the gravitational radius $R_{g}=G M / c^{2}$ (although much smaller scales were imaged in the Event Horizon Telescope observations of the central black hole in M87 [7], those observations were designed to image the shadow of the black hole, not the innermost jets). On the other hand, the frequently very well collimated jets can extend kiloparsecs, or even megaparsecs, from the galactic nucleus. Observations at multiple wavelengths can yield information about the spectral index distribution of the radio emission and Faraday rotation due to thermal material located between the emission region and the observer. Because Faraday rotation depends on the projection of the $\mathbf{B}$ field onto the line of sight to the observer, the presence of a toroidal B-field component encircling an AGN jet should produce a monotonic gradient in the Faraday rotation across the jet. This could be, for example, the toroidal component of the helical B field that is expected to form when the jets are launched (see Sections 2.1 and 2.2).

Many local effects may also contribute to the observed jet B fields, such as shocks, turbulence, jet bending and interactions with the surrounding medium. It can be challenging to discern from observations which observed features are predominantly associated with such local effects, and which reflect the helical/toroidal B fields produced when the jets are launched.

\subsection{Viewing Angles in the Rest Frame of the Jet and Observer}

For a photon emitted by a relativistic jet traveling outward from an AGN with a speed $\beta$ relative to the speed of light (i.e., $\beta=v / c$ ), the relationship between the angle along which the photon travels in the rest frame of the jet, $\theta^{\prime}$, and the angle along which it travels in the rest frame of the observer, $\theta$, is

$$
\cos \theta=\frac{\cos \theta^{\prime}+\beta}{1+\beta \cos \theta^{\prime}}
$$

where both $\theta^{\prime}$ and $\theta$ are measured relative to the jet direction. Thus, a photon emitted at right angles to the velocity of the jet in the jet's rest frame $\left(\theta^{\prime}=90^{\circ}\right)$ is observed to propagate at the angle

$$
\cos \theta=\beta \quad \sin \theta=\frac{1}{\Gamma}
$$


where $\Gamma$ is the Lorentz factor for the jet's relativistic motion, $\Gamma=1 / \sqrt{1-v^{2} / c^{2}}$. This is the well-known beaming, or boosting, of the source radiation in the forward direction. When $\Gamma$ is large, $\sin \theta$ will be small, with $\sin \theta \simeq \theta$, and

$$
\theta \simeq \frac{1}{\Gamma}
$$

In this case, all the emission radiated in the forward hemisphere is concentrated into a cone with half-angle $\theta$.

This also has important implications for relating what we observe to the view we are receiving of the jet. In the standard picture, the emission of core-dominated AGNs is highly beamed, and their jets are oriented close to our line of sight. If the angle of the jet to the line of sight is comparable to $1 / \Gamma$ (a small viewing angle in the observer's frame, since $\Gamma$ is high for a highly relativistic jet), this will be close to a viewing angle of $90^{\circ}$ in the jet rest frame. In other words, the polarization patterns that we observe for a considerable number of core-dominated AGNs probably correspond to a roughly "side-on" view of the jet, even though the jet is propagating at a small angle to the observer's line of sight. More specifically, it has been shown based on simulations for a sample of approximately 400 radio-loud AGNs [8] that the most probable viewing angle in the observer's frame is approximately $1 / 2 \Gamma$. The distribution of Lorentz factors inferred in this same study is broadly peaked between $\Gamma=5$ and $\Gamma=15$, corresponding to most probable viewing angles in the jet rest frame of roughly $40-60^{\circ}$, with most of the jet-rest-frame viewing angles lying in a wide range from $20-90^{\circ}$. All this indicates that we should consider the observed polarization patterns as being associated with jets viewed at intermediate to large angles in the jet rest frame.

\section{AGN Jets and Helical/Toroidal Magnetic Fields}

\subsection{Theoretical Expectations}

Since the earliest theoretical studies of relativistic jets, it has been suspected that the jet launching mechanism is electromagnetic in nature. In the Blandford-Payne process [9], a magnetic (B) field threads the accretion disk. The conditions are such that this field is frozen into the plasma in the disk, and so it is wound up as the disk rotates. The resulting magnetic pressure gradient creates a centrifugal force that accelerates some of the disk material outward along the magnetic field lines. The Blandford-Znajek mechanism [10] essentially acts to extract the rotational energy of the black hole and transform it into the electromagnetic energy of an outgoing Poynting flux that pushes jets of electrons and positrons outward. Both of these mechanisms rely on the presence of an initial magnetic field with a longitudinal compenent that is wound up by the rotation of the central region of the AGN. A toroidal component is added by the rotation, giving rise to an overall helical $\mathbf{B}$ field that propagates outward together with the jets. Examples of theoretical studies of the electromagnetic launching and subsequent outward propagation of jets together with the helical fields that are produced include the relatively early studies of Nakamura et al. [11] and Lovelace et al. [12], and the more recent simulations of Tchekovskoy and Bromberg [13] and Barniol Duran et al. [14].

Although it is not possible to image the scales on which the jets are launched directly, intriguing evidence that the jet production mechanism is electromagnetic is provided by the study of Zamaninasab et al. [15]. These authors found a tight correlation between the accretion-disk luminosities estimated from optical-line observations, taken to be a proxy for the magnetic flux near the black hole, and the magnetic fluxes measured in their VLBI jets, estimated from the frequency dependence of the VLBI core position and the jet cross sections. This demonstrates that the magnetic flux near the black hole is proportional to the magnetic flux far down the jets, as is expected if the jets are indeed produced by the Blandford-Znajek mechanism. It is also remarkable that the derived numerical proportionality between the accretion-disk luminosity and the jet magnetic flux is the value predicted for a magnetically arrested accretion disk (MAD), strongly suggesting that the 
MAD scenario described by Tchekhovskoy et al. [16] and McKinney et al. [17] is operating in radio-loud AGNs.

In practice, it is most likely that both mechanisms operate-the Blandford-Znajek mechanism near the jet axis and the Blandford-Payne mechanism somewhat farther out in the accretion disk. The regions influenced by the two mechanisms should each carry related helical $\mathbf{B}$ fields that are produced by the rotation of the central black hole and accretion disk. The mechanism or mechanisms collimating the jets are not entirely clear, and it is possible that the toroidal component of this helical field plays a role. The direction of the initial longitudinal B-field component that is wound up (inward or outward) is often taken to random. However, it may be that it has a preferred orientation, as in the "Cosmic Battery" model of Contopoulos et al. [18] and Christodoulou et al. [19].

The presence of a toroidal $\mathbf{B}$-field component requires that there be a current in the jet, with the direction of this current determined by the right-hand rule (see Figure 1); thus, studies of helical jet $\mathbf{B}$ fields are inherently also concerned with the currents flowing in AGN jets, and the mechanisms giving rise to these currents.

It is important to note that the presence of a helical or toroidal B-field component is not meant to suggest that the magnetic field is perfectly ordered. As is mentioned above, typical values for the degree of linear polarization in the optically thin jet, $\simeq 5-15 \%$, directly demonstrate that there is also an appreciable disordered (tangled) component of the field. Some studies suggest that this combination of helical and disordered field components can explain both the observed polarization structures and rapid polarization variability, with perhaps up to $30-50 \%$ of the overall field being in the ordered component (e.g., [20]).

\subsection{Faraday Rotation Gradients}

An electromagnetic wave can be described as the sum of any two mutually orthogonal components of the wave's electrc (E) field, such as its right circularly polarized (RCP) and left circularly polarized (LCP) components. When an electromagnetic wave passes through a region with magnetic field and free electrons (a magnetized plasma) along the path from the source to the observer, the free electrons in the plasma interact slightly differently with the RCP and LCP components of the polarized wave, leading to different speeds of propagation for these two components in the medium. As the polarized wave traverses the magnetized medium, the RCP and LCP components become increasingly out of synch, leading to Faraday rotation of the wave's plane of polarization. The rotation of the EVPAs depends on the $\mathbf{B}$ field in the region of Faraday rotation, the number density of the charged particles bringing about the Faraday rotation $n$, their charge $e$ and mass $m$, and the wavelength of the radiation $\lambda$ :

$$
\chi=\chi_{o}+R M \lambda^{2} \quad R M=\frac{e^{3}}{8 \pi^{2} \epsilon_{o} m^{2} c^{3}} \int n \mathbf{B} \cdot d \mathbf{l}
$$

where $\chi_{0}$ is the emitted EVPA, $\chi$ the observed EVPA, RM the Faraday rotaton measure, $\epsilon_{0}$ the permittivity of free space, and $c$ the speed of light in vacuum. The integral is carried out over the entire line of sight from the source to the observer.

Due to the $1 / \mathrm{m}^{2}$ dependence in the $\mathrm{RM}$, it is usually assumed that the charged particles producing the observed RM are electrons, rather than protons. Similarly, because relativistic electrons have appreciably higher effective masses than non-relativistic ones, it is usually assumed that the electrons producing the Faraday rotation are non-relativistic (thermal). A plasma containing electrons and positrons with equal densities would not give rise to any net Faraday rotation, as the signs of the Faraday rotation produced by the electrons and positrons would be opposite.

The RM depends on the electron density, the line-of-sight $\mathbf{B}$ field and the path length through the magnetized plasma. The only one of these factors that affects the sign of the RM is the line-of-sight B field, i.e., whether it is directed toward (positive) or away from (negative) the observer. This means that the sign of the RM reflects the direction of the line-of-sight $\mathbf{B}$ field, effectively adding a third dimension to the $\mathbf{B}$-field structure 
projected onto the plane of the sky, provided by the linear-polarization distribution. When the Faraday rotation arises outside the region of emission in the source, the observed EVPA $\chi$ depends linearly on $\lambda^{2}$, as is indicated above. Deviations from a linear $\lambda^{2}$ dependence can arise if multiple regions with different intrinsic polarizations and undergoing different Faraday rotations are unresolved in a particular set of observations; in this case, fitting of models to the observed Stokes parameters $Q$ and $U$ may be required to deduce the properties of these regions (e.g., [21]).
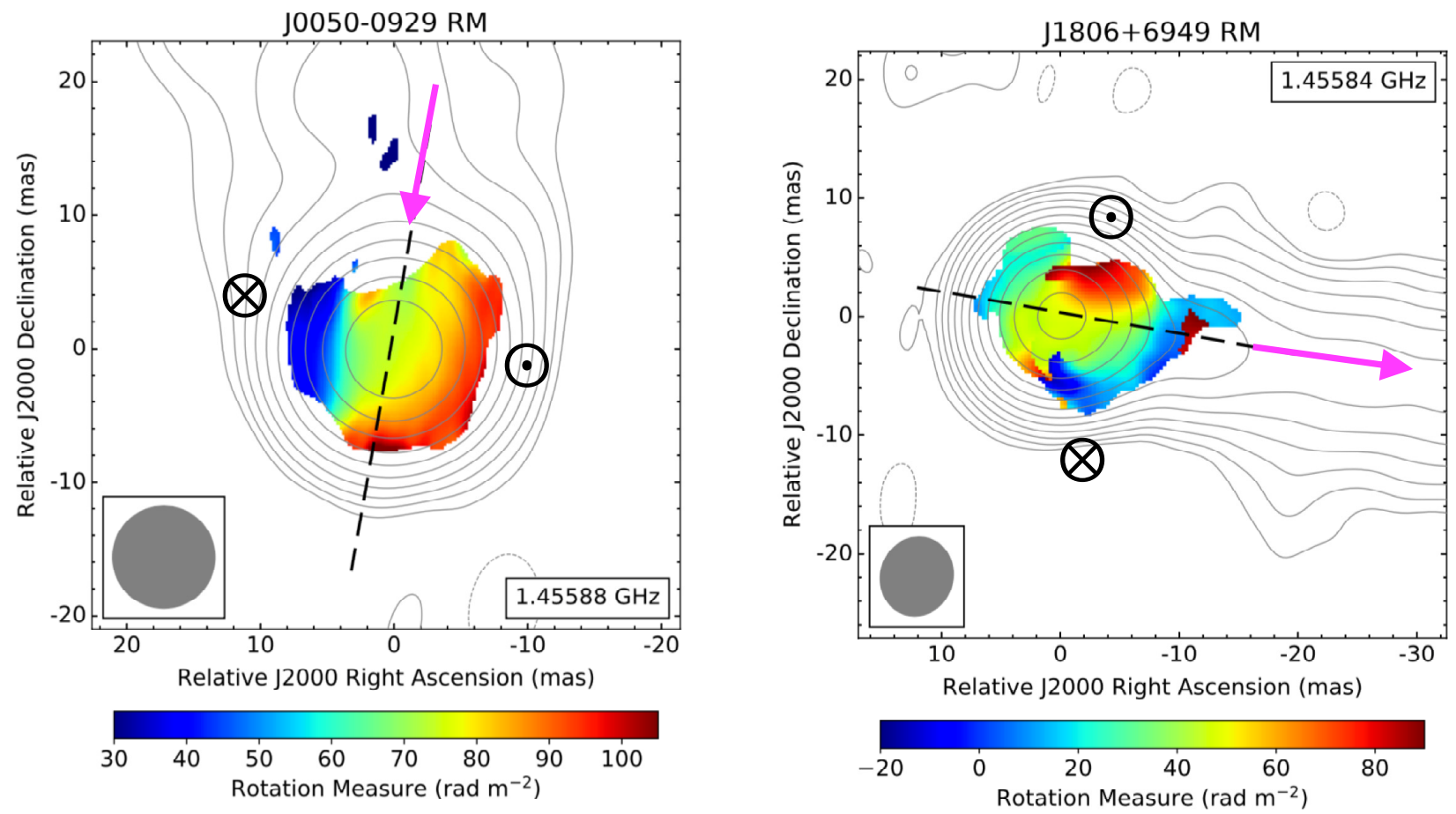

Figure 1. Two examples of transverse Faraday rotation (RM) gradients detected across parsec-scale core-jet structures. The circled dots and crosses indicate the sides of the jet where the toroidal B-field component is oriented toward and away from the observer, respectively. The pink arrows show the inferred direction of the current along the local jet axis, shown by the dashed line. The sizes of the Gaussian restoring beams are shown by the ellipses in the bottom left-hand corners of the images. The RM maps used to make this figure were made and provided by Frederick Richardson.

If a jet and the ambient thermal plasma carry a helical $\mathbf{B}$ field, the projection of the toroidal component of this field onto the line of sight changes monotonically across the jet. This should give rise to a corresponding gradient in the Faraday rotation across the jet, and the detection of such a transverse RM gradient provides a direct diagnostic of the presence of a toroidal B-field component. This idea was first considered by Perley et al. [22] and Blandford [23] in the 1980s and 1990s, but it was overlooked until the detection of a clear RM gradient across the VLBI jet of 3C273 by Asada et al. [24]. Two examples detected recently using VLBA observations made simultaneously at 6, 13, 18 and $22 \mathrm{~cm}$ are shown in Figure 1.

Statistically significant $(\geq 3 \sigma)$ transverse RM gradients have been detected across the jets of some 50 AGNs on parsec scales [25]. In addition, such gradients have been detected across the kiloparsec-scale jets of a number of AGNs and radio galaxies [19,26,27]. The trends shown by these collected results are discussed in Section 2.3.

Note that it was stated without demonstration in [28] that, in order to be considered reliable, a transverse RM gradient must encompass at least three beam widths, and must not be located in a partially optically thick region such as the core. However, the Monte Carlo simulations of [29] have shown that this restriction on the width of a reliable transverse RM gradient is inappropriate-simulated transverse RM gradients were detectable even in cases where the intrinsic jet width was much less than a beam width. In addition, as was pointed 
out above, the results of [4] imply that virtually all the VLBA-scale polarized emission observed in AGNs arises in optically thin regions, including polarization from the core region. Therefore, only the remaining two constraints stated in [28] are appropriate: that the overall change in the RM be at least three times the typical error (i.e., that the magnitude of the gradient exceed $3 \sigma$ ), and that the change in the RM across the jet be monotonically smooth within the errors. These last two criteria have both been implemented in subsequent searches for statistically significant transverse RM gradients ([25] and references therein).

\subsection{Global Patterns in the B-Fields and Currents of AGN Jets}

The direction of an RM gradient observed across the jet of an AGN is determined by the direction of the toroidal B-field component of the jet, which, in turn, determines the direction for the current flowing along the jet axis, in accordance with the usual righthand rule. The directions for the toroidal fields and currents implied by the two observed transverse RM gradients in Figure 1 are also shown in that figure. The direction of the toroidal component of the jet's helical $\mathbf{B}$ field is determined by the direction of the rotation of the central black hole and its accretion disk and the direction of the initial longitudinal field component that is wound up by the rotation.

Studies considering time variability of the observed parsec-scale Faraday rotation include [30-35]. In general, time variability of the observed RM values and their distribution could be due to variability in the thermal electron content and/or magnetic-field structure in the region of Faraday rotation, as well as changes in the thickness of this region with time. Dramatic time variability in the parsec-scale RM distribution in 3C273, with, however, a persistent transverse RM gradient, has been known to be present for more than 20 years [30,31]. This has recently been explained by Lisakov et al. [35] as a consequence of the motion of the jet within a much broader cone than is visible at any one epoch. In this picture, a helical $\mathbf{B}$ field is embedded in this larger region surrounding the jet, explaining the persistent transverse RM gradient, but the jet emission passes through different parts of this region as its orientation changes, giving rise to dramatically different RM magnitudes at different epochs.

In the simplest picture, we would expect the toroidal component of the jet $\mathbf{B}$ field, and therefore the associated observed transverse RM gradient, to have the same direction all along the jet and to be consisent in time. This has indeed been observed in some AGN (see [25] and references therein), indicating that these structures are fairly stable. Surprisingly, however, reversals in the directions of observed transverse RM gradients across the parsec-scale jets are also sometimes observed, either with distance from the VLBI core or with time (see [25] and references therein). Two examples are shown in Figure 2. This behaviour can be explained if there are two regions of helical $\mathbf{B}$ field surrounding the jet, one inside the other, and with the directions of the toroidal field components being opposite in the two regions $[29,32]$. The key factor here is that the line of sight from the emission region to the observer passes through both of these regions of helical $\mathbf{B}$ field, and each makes a contribution ot the overall observed Faraday rotation. Since the directions of the toroidal field components are opposite in the two regions, their contributions partially cancel each other out; the direction of the observed transverse RM gradient depends on whether the inner or the outer region makes the larger contribution to the total observed Faraday rotation. It seems natural to suppose that the relative contributions of these two regions of helical $\mathbf{B}$ could change as the electron density and magnetic field vary with distance from the jet base, leading to a reversal in the direction of the observed transverse RM gradients at the distance where predominance of one makes a transition to predominance of the other. Similarly, if changing conditions in and around the jet with time lead to a change in whether the inner or outer region of helical field dominates the total Faraday rotation, this could lead to corresponding changes in the direction of the resulting transverse RM gradient with time.

In fact, just such a nested helical-field configuration is expected in the "cosmic battery" model of [18,19], as well as for a dipole-like B field that is wound up by rotation (see 
Section 2.6). The "cosmic battery" model predicts that the toroidal component of the inner helical field should correspond to an inward current along the jet axis, while the toroidal component of the outer helical field should correspond to an outward current farther from the jet axis, with this latter current being present between the inner and outer regions of helical field. This situation is shown schematically in Figure 2 in [25].
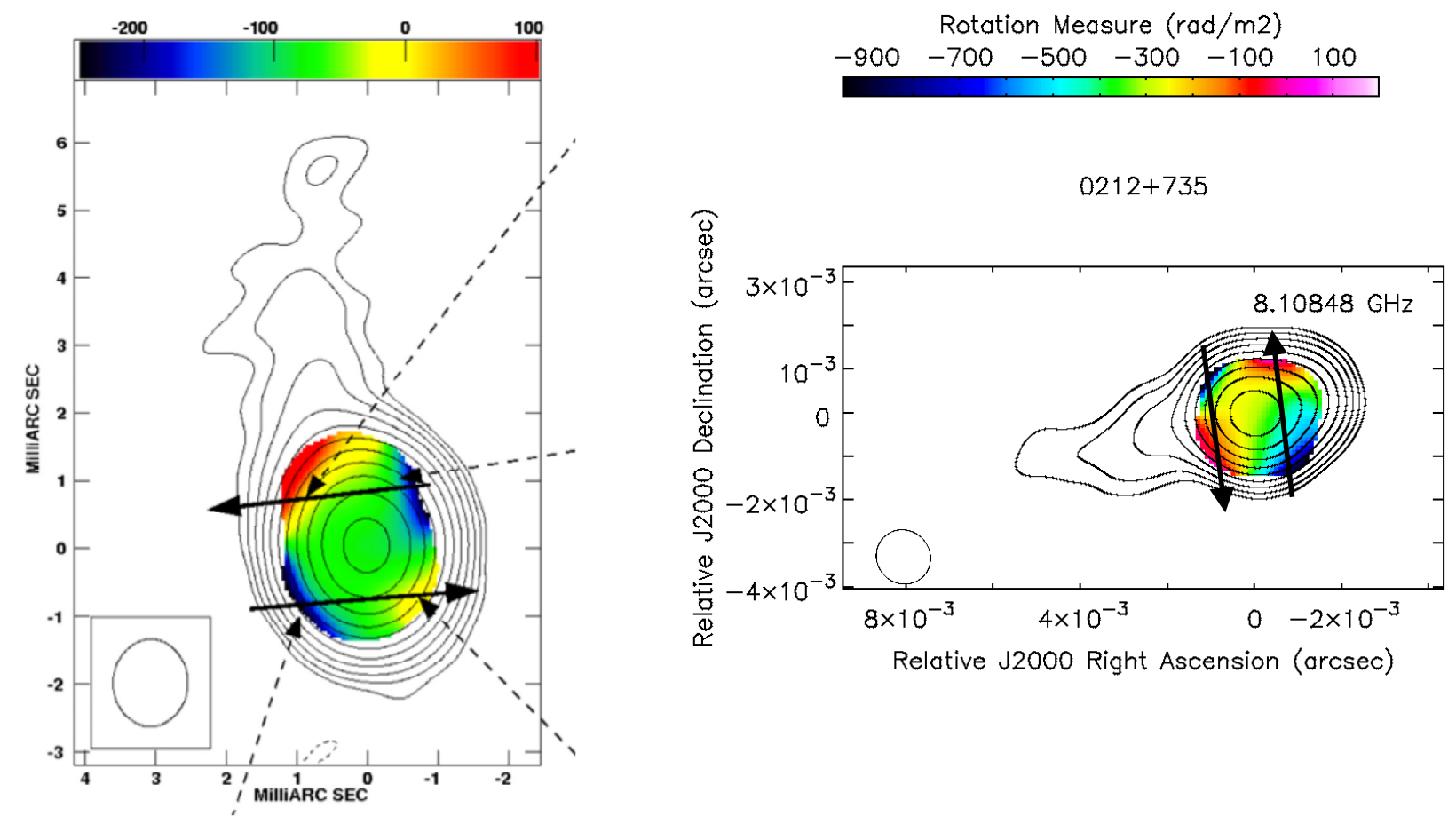

Figure 2. Two examples of reversals in the directions of transverse RM gradients observed across the core and inner jet. The left part of the figure shows the RM map of 0716+714, adapted from Figure 3 of [29], and the right part of the figure the RM map of $0212+735$, adapted from [25]. The sizes of the Gaussian restoring beams are shown by the ellipses in the bottom left-hand corners of the images.

After considerable efforts to search for reliable transverse RM gradients across AGN jets, sufficient statistics to test this picture are now available. Transverse RM gradients have now been detected across about 50 AGN jets imaged on parsec scales with VLBI. The collected results of $[25,36]$ indicate a preference for inward currents, with the probability of this preference coming about by chance being $\simeq 0.20 \%$. In contrast, the combined results of $[19,26,27]$, show a strong preference for outward currents on decaparsec-kiloparsec scales, with the probability of this preference coming about by chance being $\simeq 0.36 \%$. These observations are consistent with the nested helical-field configuration described above. The currents associated with the two regions of helical field form huge loops oriented roughly perpendicular to the accretion disk. Currents flowing inward along the jet axis and outward in an extended region around the jet are closed by currents flowing away from the jet axis in the plane of the accretion disk and back toward the jet axis in a region near the end of the jet, in a quadrupolar-like pattern.

Determining the physical origin of this set of $\mathbf{B}$ fields and currents is crucial for our understanding of the launching and propagation of AGN jets. The presence of a net current along the jet axis implies different velocities for the positive and negative charges in the jet plasma. It is interesting in this connection that Plavin et al. [37,38] and Hovatta et al. [39] have recently argued that AGNs are likely sources of astrophysical neutrinos, and that this may, in turn, imply the presence of a significant admixture of highly energetic protons in the jet material. One possible origin for an inward jet current could be a slightly slower outflow velocity for the jet protons, compared to the jet electrons, but this is purely speculative at the moment. 
Thus, the collected data suggest a nested helical-field configuration, with inward current near the jet axis and outward current in a more extended region around the jet. Any physical model for the overall B-field structure of AGN jets must be able to explain this configuration, and provide reasonable physical origins for a predominance of AGN jets with inward current along their jet axes.

\subsection{What about Other Explanations for Observed Transverse RM Gradients?}

In principle, a transverse RM gradient could come about because of a corresponding gradient in the thermal electron density in the vicinity of the jet. However, variations in the electron density cannot affect the sign of the Faraday rotation measure, which is determined solely by the direction of the $\mathbf{B}$ field along the line of sight. This means that a transverse RM gradient due to a toroidal B-field component can potentially run from negative to positive RMs. It is important in this connection that a significant fraction of the transverse RM gradients that have been detected across AGN jets change sign from one side of the jet to the other; for example, 18 of 39 transverse RM gradients reported in $[25,36,40]$ encompassed RM values of both signs (approximately 50\%). At the same time, a transverse RM gradient produced to a helical field may encompass RM values of only one sign for some helical-field pitch angles and jet viewing angles. Thus, the lack of a sign change in the RM across the jet does not argue against an RM gradient being produced by a helical jet $\mathbf{B}$ field. In addition, it would not be not physically plausible for electron-density gradients to display a preferred direction across the jet-for example, from north to south; however, this asymmetry arises in a natural way if the current in the jet has a preferred direction-inward or outward-since this gives rise to a corresponding preferred direction for the toroidal B-field in the jet.

Although the magnitudes of the rotation measures will be affected by the local Doppler factor, it is difficult to imagine a physically plausible picture in which this to leads to systematic gradients in the observed RM across the entire jet. The reason for this is that the Doppler factor depends on the intrinsic velocity of the jet outflow and the angle at which the jet is viewed, and these are not expected to change systematically across the jet.

\subsection{Helical vs. Toroidal Fields}

The detection of a transverse RM gradient indicates the presence of a toroidal $\mathbf{B}$-field component, and this will be associated with an overall helical B field only if the field also has a longitudinal component. It is therefore important to identify ways in which we can distinguish between a purely toroidal $\mathbf{B}$ field or a toroidal field that represents one component of a helical B field. The key to this is asymmetries in the intensity and polarization profiles across the jet.

As is shown in Figure 3, a helical field that is viewed at an angle other than $90^{\circ}$ to the jet axis has an ordered longitudinal component, which should give rise to asymmetric profiles of the intensity, linear polarization and Faraday rotation across the jet (upper part of Figure 3). The predominant $\mathbf{B}$ field along the upper edge of the helical field in the upper part of Figure 3 lies close to the line of sight, leading to relatively lower intensities and degrees of polarization and higher rotation measures. On the contrary, the predominant B field along the lower edge of the helical field in the upper part of Figure 3 lies close to the plane of the sky, leading to relatively higher intensitie and degrees of polarization and lower RM values. These patterns come about because the synchrotron intensity and degree of polarization depend on the projection of the $\mathbf{B}$ field onto the plane of the sky, while the magnitude of the Faraday rotation depends on the projection of the $\mathbf{B}$ field onto the line of sight.

In contrast, a helical field viewed exactly at $90^{\circ}$ to the jet axis or a toroidal field viewed at any angle to the jet axis should give rise to symmetric intensity, polarization and RM profiles across the jet (lower part of Figure 3; see, e.g., [25,41]).

We must also take into account aberration when considering at what angle we view the jet in the jet rest frame (Section 1.2): a small viewing angle in the observer's frame, 
comparable to $1 / \Gamma$, where $\Gamma$ is the Lorentz factor for the bulk motion of the jet, will be close to a viewing angle of $90^{\circ}$ in the jet rest frame. This means that the situations depicted in Figure 3 (viewing the jet precisely or roughly "from the side") are relevant here.

The prediction of an anti-correlation between the side of the jet with higher degrees of polarization and the side with higher RM magnitudes can potentially provide an interesting consistency test of whether transverse Faraday RM gradients result from helical jet $\mathbf{B}$ fields. A small number of cases where this pattern may be observed have been noted (e.g., [41]). However, it is often not straightforward to know how to correctly compare the degree of polarization and RM values measured on the two sides of the jet when the jet is curved or the distance from the core where the side-to-side difference in the degree of polarization is clearest is different from the distance where the transverse RM gradient is clearest. This is nevertheless a potentially fruitful area of research, and is worthy of further study using larger samples of AGNs.

\section{B field close to line of sight Higher RM, lower degree of polarization

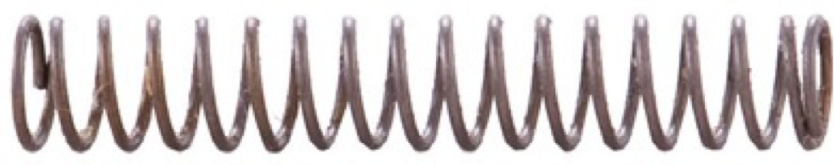 \\ $B$ field close to plane of the sky Lower RM, higher degree of polarization}

\section{amomommom ARARA}

\section{Symmetrical transverse Faraday rotation and degree of polarization structure}

Figure 3. Schematic illustration of the origin of the asymmetric polarization and RM patterns expected for a helical B field observed at an angle other than $90^{\circ}$ to the jet axis (top) and the symmetric polarization and RM patterns expected for a purely toroidal field viewed at any angle or a helical field viewed at precisely $90^{\circ}$ to the jet axis (bottom).

\subsection{The Initial Field That Is Wound Up—Dipolar or Quadrupolar?}

McKinney and Blandford [42] used large-scale general relativistic magnetohydrodynamic simulations of rapidly rotating, accreting black holes producing jets to investigate the stability of the jet formation process during the accretion of initially dipolar (left part of Figure 4) and quadrupolar (right part of Figure 4) fields. They found that, in the dipolar case, jets were produced which were able to achieve high bulk Lorentz factors without significant disruption as they propagated. On the contrary, the accretion of a quadrupolar field did not produce steady relativistic jets. This leads to the prediction that the accreted $\mathbf{B}$ field has a dipolar character, since steady relativistic jets do form and propagate outward in AGNs.

It is of interest to test this theoretical prediction by checking to see whether the transverse Faraday rotation gradients for the jet and counter jets show the signatures of a initially dipolar or a quadrupolar field. The patterns displayed by the toroidal B-field components produced by the rotation of the central black hole and accretion disk in these 
two cases are shown in Figure 5. The initial dipolar-type B-field configuration gives rise to oppositely directed RM gradients across the two jets, projected onto the sky, while the initial quadrupolar-like configuration gives rise to similarly directed RM gradients. Note that the dipolar-like configuration appears the same if the image is rotated by $180^{\circ}$, while the quadrupolar-like configuration does not.
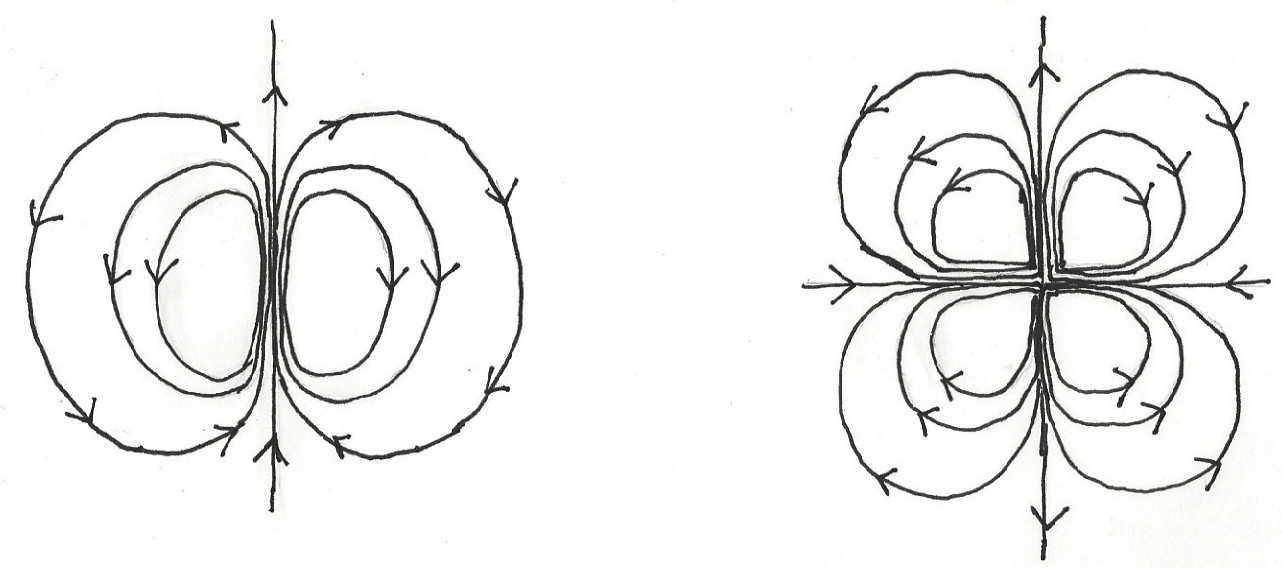

Figure 4. Schematic diagrams of dipolar (left) and quadrupolar (right) B-field configurations.

Thus, observations of the patterns shown by the transverse RM gradients across the two oppositely-directed jets emerging from the central regions of AGNs can provide information about whether the initial B-field configurations that are accreted are dipolar like or quadrupolar like. As the counterjets of core-dominated AGNs are almost always undetected due to the effects of Doppler boosting, the most promising place to try to obtain such observations is two-sided jets on kiloparsec scales. Thus far, transverse RM gradients have been firmly detected across both kiloparsec-scale jets of two radio galaxies: 5C4.114 ([43], Figure 6, left) and IC4296 [27]. In the case of a third radio galaxy, Coma A (3C 277.3), one transverse RM gradient is firm, but the other is tentative ([44], Figure 6, right). In all three cases, the pattern shown corresponds to a dipolar-type field, but more cases must be found before we will know whether this is typical.

We can draw some further deductions based on the current distributions expected for the two configurations. Application of the right-hand rule shows that the wound-up dipolar configuration in the upper part of Figure 5 corresponds to inward currents along the jet axes; winding up the same dipolar configuration by rotation in the opposite direction would reverse the toroidal field directions for both jets, yielding outward currents along both jet axes. In contrast, the wound up quadrupolar configuration shown in the lower part of Figure 5 corresponds to an inward current in the left-hand jet and an outward current in the right-hand jet; again, winding up the same quadrupolar configuration by rotation in the opposite direction would reverse the toroidal field directions for both jets, and thus reverse the axial current directions along both jets. Therefore, if a predominance of inward or outward currents is observed for AGN jets, this implies a dipolar initial field configuration, as there is no way to get a statistical imbalance between inward and outward currents in the case of a quadrupolar initial field. Since a statistically significant predominance of inward currents has been observed for the parsec-scale jets of AGNs (see Section 2.3), this supports the hypothesis that the initial field configuration is dipolar like. Another point against the possibility that the fields accreted could be quadrupolar is that it doesn't seem reasonable that the jet and counterjet—believed to be essentially identical—would have different current directions (inward and outward), implying inherently different physical conditions. 


\section{Initial Dipolar-type B field Wound up by Rotation}

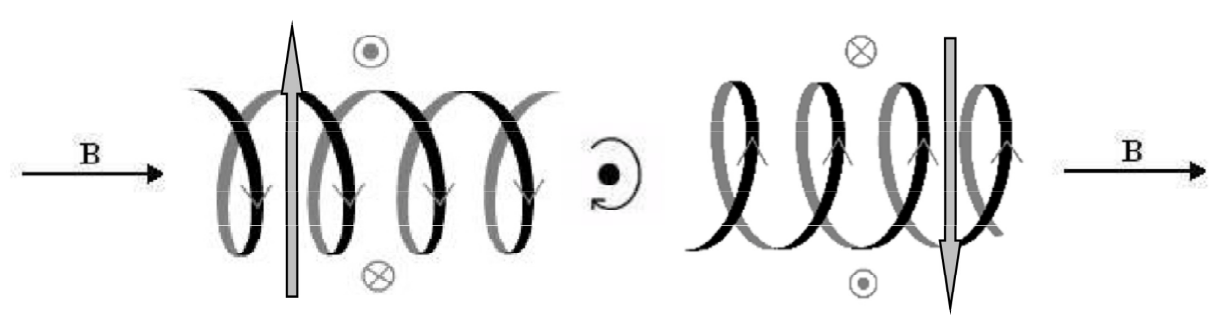

Initial Quadrupolar-type B field Wound up by Rotation
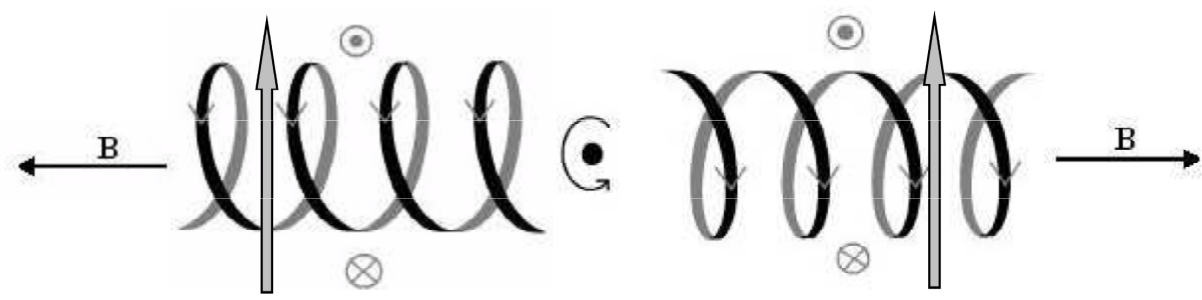

Figure 5. Illustration of the toroidal B-field components arising when the parts of the dipolar and quadrupolar fields near the axis are wound up by rotation of the central black hole. The arrows labeled " $\mathrm{B}$ " indicate the initial axial B-field directions before being wound up, the circles with an $\mathrm{x}$ or dot inside indicate the direction of the resulting toroidal field, into and out of the page, respectively. The gray arrows indicate the direction of the resulting systematic Faraday rotation gradient across the jet due to the toroidal field component.
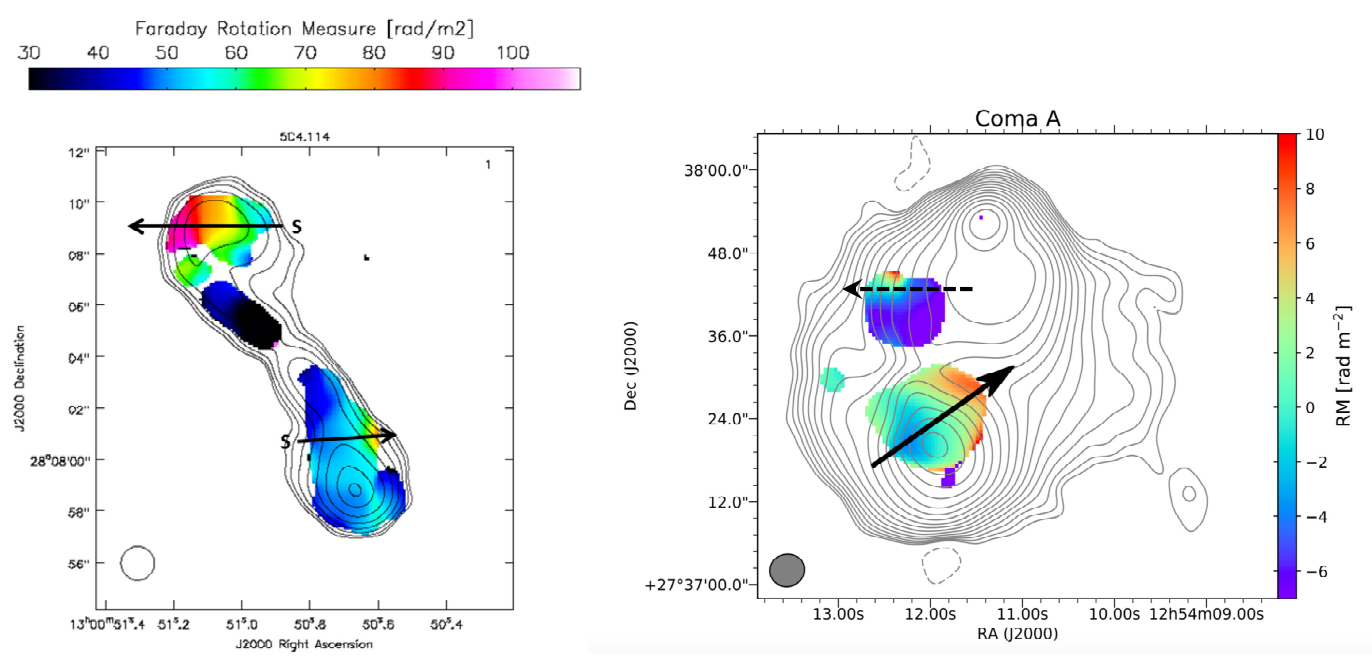

Figure 6. Transverse RM gradients across the two jets of the radio galaxies 5C4.114 (left, adapted from [43]) and Coma A (right, adapted with permission from [27]), showing the pattern expected for winding up an initially dipolar B field (upper part of Figure 5). The black arrows across the two jets highlight the directions for the corresponding transverse RM gradients. Both transverse RM gradients in 5C4.114 and the southern transverse RM gradient in Coma A have significances exceeding $3 \sigma$; the significance of the northern gradient in Coma A is less than $3 \sigma$, and must be considered tentative. The sizes of the Gaussian restoring beams are shown by the ellipses in the bottom left-hand corners of the images.

Finally, the schematics in Figure 5 only shown the result of winding up the part of the dipolar or quadrupolar fields near the jet axis. The fields farther away from the jet axis will be oppositely directed, and will give rise to oppositely directed transverse RM gradients and currents. The overall system of currents for the dipolar-type field is quadrupolar like, and the overall system of currents for the quadrupolar-type field is dipolar like 
(you can verify this by applying the right-hand rule). Thus, an initial dipolar-like B-field configuration should give rise to a nested $\mathbf{B}$-field structure such as the one described in Section 2.3. The interesting thing is to discern why the direction of rotation of the central black hole and its accretion disk is coupled with the direction of the $\mathbf{B}$ field that is wound up, in such a way that this gives rise to a predominance of inward currents near the jet axis and outward currents farther from the axis. The model of $[18,19]$ can do this in a natural way, but other physical models may also be possible.

\section{Characteristic Magnetic Field Structures of AGN Jets-Global or Local Origin? \\ 3.1. Polarization Structures}

The observed parsec-scale EVPAs in the jets of AGNs often tend to lie either parallel to or perpendicular to the local jet direction (when the influence of Faraday rotation is not large). Since the associated jet B field is orthogonal to the EVPAs in optically thin regions, and virtually all the observed polarization arises in optically thin regions [4], this implies that the jet $\mathbf{B}$ field also tends to be oriented parallel or perpendicular to the local jet direction. This tendency has long been known, and can be seen in virtually all published VLBI images that are not appreciably influenced by Faraday rotation, such as the collected $2 \mathrm{~cm}$ VLBI polarization images for the MOJAVE project (e.g., [2]). Lyutikov et al. [45] have noted that this comes about naturally if the small sections of the jets that are observed in a single image display local cylindrical symmetry, since the $\mathbf{B}$ field can in that case always be described as having components along and perpendicular to the jet projected onto the sky.

The tendency for the observed polarization to lie parallel or perpendicular to the local jet direction can give rise to a rich array of observed polarization (B-field) structures, depending on whether a region of polarization is localized or present over extensive regions of the jet, and whether specific localized regions of polarization are observed across the jet, only along the "spine" of the jet, or only at one or both of the jet edges. A schematic of various polarization configurations commonly observed is given in [46].

Helical jet $\mathbf{B}$ fields could explain basically all of the polarization patterns that are observed. For example, if the pitch angle of a helical jet $\mathbf{B}$ field is low, this will tend to rise to predominantly longitudinal $\mathbf{B}$ fields, while those with high pitch angles will tend to give rise to predominantly orthogonal $\mathbf{B}$ fields, relative to the jet direction. Fairly complex patterns such as a "spine-sheath" transverse polarization structure, with perpendicular $\mathbf{B}$ near the jet ridge line and longitudinal $\mathbf{B}$ field at the jet edges, can also be explained by a helical jet $\mathbf{B}$ field, if the toroidal component dominates near the jet axis, while the longitudinal component dominates near the jet edges (e.g., [45,47]). Depending on the pitch angle of the helical field and the viewing angle of the jet, and the sensitivity of the observations, situations are also possible in which longitudinal and orthogonal $\mathbf{B}$ fields predominate on either side of the jet, or longitudinal $\mathbf{B}$ field predominates on one side of the jet only (see, e.g., [41]).

The pitch angle of the helical field carried by an AGN jet should depend in part on the ratio of the velocities of the rotation and outflow. It has independently been suggested in $[24,48]$ that the, on average, lower typical superluminal speeds of BL Lac objects compared to those of quasars may be related to the predominance of $\mathbf{B}$ fields that are orthogonal to/aligned with the jet direction in the VLBI jets of BL Lac objects/quasars. If the intrinsic outflow speeds of quasars are typically higher than those of BL Lac objects, this could lead to lower pitch angles for their helical jet $\mathbf{B}$ fields, and so a higher tendency for the predominant $\mathbf{B}$ field component to be longitudinal.

An interesting potential source of longitudinal $\mathbf{B}$ field is the enhancement of an initial longitudinal field component (possibly but not necessarily associated with a helical B field) at the outer part of a bend in the jet - the $\mathbf{B}$ field at the outer edge of the bent jet is stretched along the flow, enhancing the longitudinal component. An example of an AGN jet in which this may be happening is shown in Figure 7. 


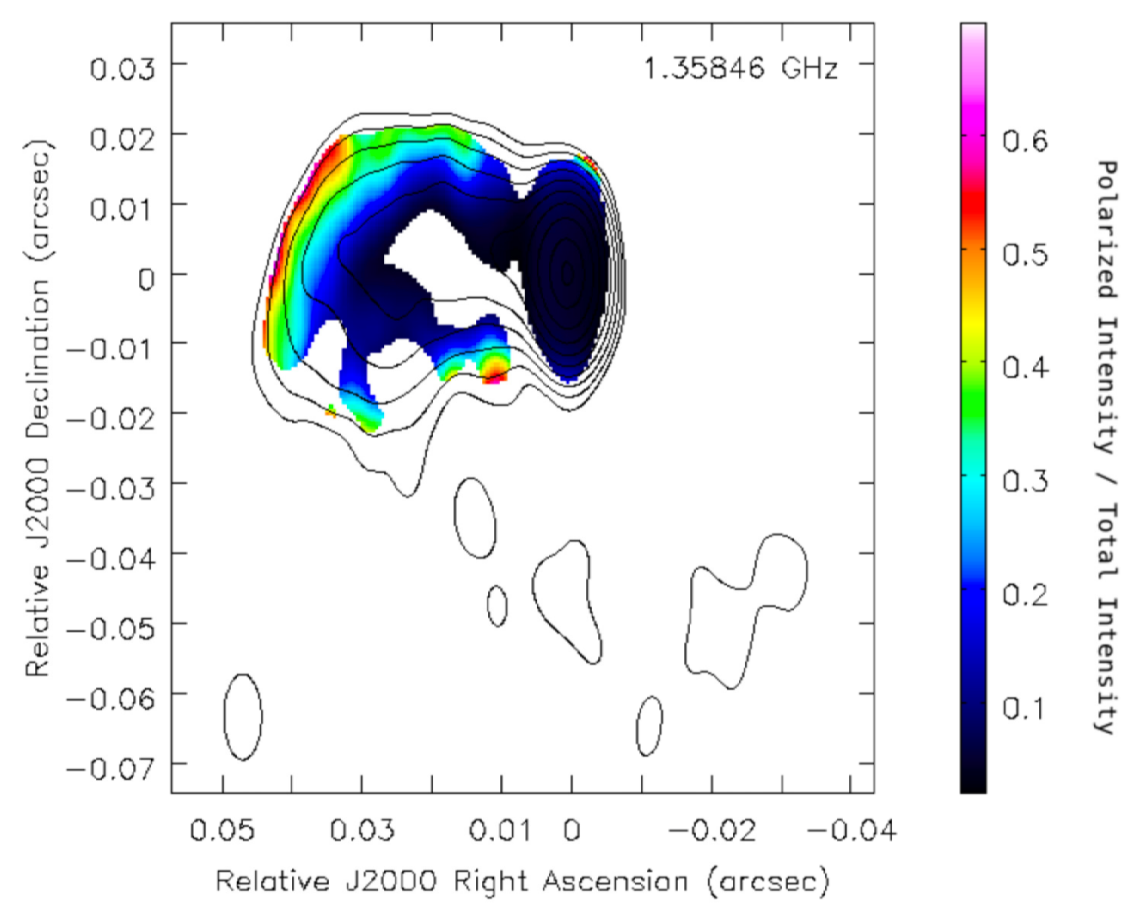

Figure 7. Possible curvature-induced polarization at the outer edge of a bend in the jet of J0738+1742. The EVPAs (not shown here) indicate a longitudinal B field along the outer edge of the curved jet and the fractional polarization rises toward the outer edge of the bend. The size of the Gaussian restoring beam is shown by the ellipse in the bottom left-hand corner of the image. The map is reproduced with permission from [49].

Regions of orthogonal $\mathbf{B}$ field concentrated in bright, compact features are not naturally explained as being associated with helical jet $\mathbf{B}$ fields. This behaviour is most naturally explained as a result of shock compression, which enhances the orthogonal component of the field (see, e.g., [50-53]). Shocks are believed to play an important role in variability of the jet emission, especially polarization variability.

In the absence of other evidence for the presence of a helical $\mathbf{B}$ field, such as a transverse RM gradient, regions of longitudinal $\mathbf{B}$ field (orthogonal linear polarization) at the edge of the jet can also be naturally explained as a result of shear interactions with the ambient medium through which the jet propagates (e.g., [54] and references therein). It is difficult to identify the most likely origin of such polarization (a helical field or shear) without some other information. Clausen-Brown et al. [55] explore transverse asymmetries in the intensity, fractional polarization, Faraday rotation and spectral index that could arise when the jet carries a helical B field. Unfortunately, some of this transverse structure, such as an increase in fractional polarization accompanied by a flattening of the spectral index at one edge of the jet, could come about due to either interactions with an ambient medium or a helical jet B field. Further theoretical studies or simulations identifying properties that could distinguish between these two origins would be valuable.

Some of the complex observed polarization patterns that could arise due to the presence of a helical jet $\mathbf{B}$ field could also be explained by the joint action of multiple local phenomena, such as shocks and interactions with the ambient medium (see, e.g., [56]). Here, we should recall the principle of Occam's razor, which states that "if more than one explanation is possible, the simplest should be preferred". In other words, the more assumptions that must be invoked to explain the observations using a specified model, the less likely that this is the correct explanation. Since helical jet $\mathbf{B}$ fields are expected to be present on firm theoretical grounds, Occam's razor suggests we should first consider whether a helical jet $\mathbf{B}$ can provide a natural explanation for some aspects of the observed polarization structure, then look to other factors such as shocks, shear and curvature of 
the jet to explain localized features that cannot be easily explained by a helical jet $\mathbf{B}$ field. Ideally, supplementary information, such as spectral data or modeling results should also be incorporated when possible. This approach can provide the simplest and also fullest picture of the jet and the actions of various local factors.

\subsection{Limb Brightening}

Dramatic limb brightening has been observed in the jets of a number of relatively nearby AGNs. Three examples are given in Figures 8 and 9. Most recently, prominent limb brightening has been detected for the parsec-scale jet and counterjet of Cen A [6]. There is a growing suspicion that this could be a common feature of AGN jets, but its origin is not clear. Proposed explanations for limb brighening include mass loading at the edges of the jet; Kelvin-Helmholtz instability, magnetic reconnection and particle acceleration at the edges of the jet (e.g., [57]); a velocity difference between the central spine and a slower sheath around jet, with the sheath emission preferentially beamed toward the observer; and a helical or toroidal field carried by the jet-the synchrotron emissivity is greatest when the projected $\mathbf{B}$ field is in plane of the sky, which arises at the jet edges. As an example, Figure 10 shows relativistic magnetohydrodynamical (RMHD) computations of the intensity distributions produced by jets carrying helical and toroidal $\mathbf{B}$ fields, carried out by Kramer and MacDonald [58]. Takahashi et al. [59] have argued that the relatively symmetrical brightness of observed limb-brightened jets is consistent with these jets produced by the Blandford-Znajek mechanism operating in a rapidly spinning black hole.

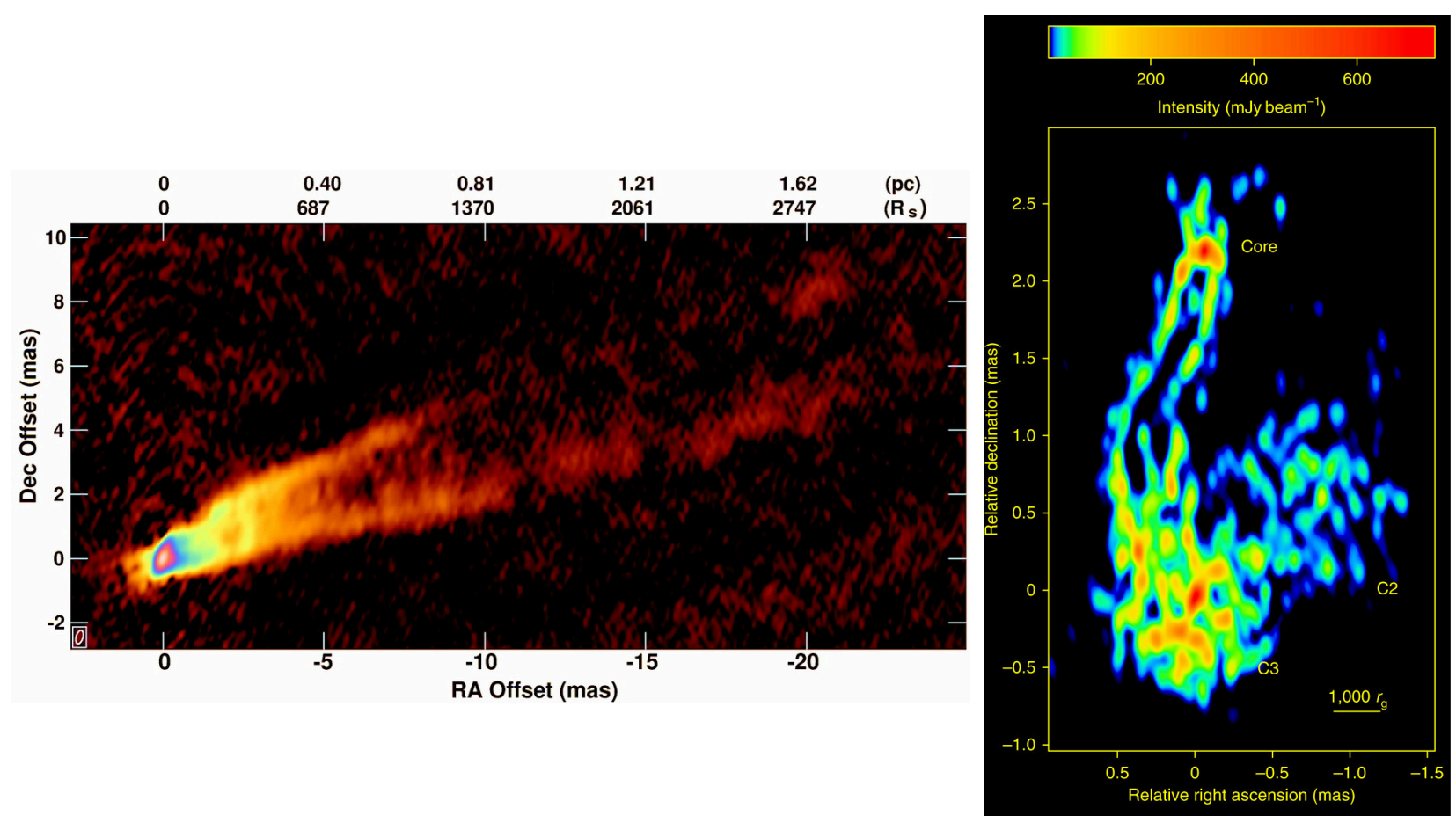

Figure 8. Two examples of AGN jets displaying prominent limb brightening: M87 (left, reprinted with permission from [60]; copyright 2018 American Astronomical Society) and 3C84 (right, reproduced with permission from [61]). 


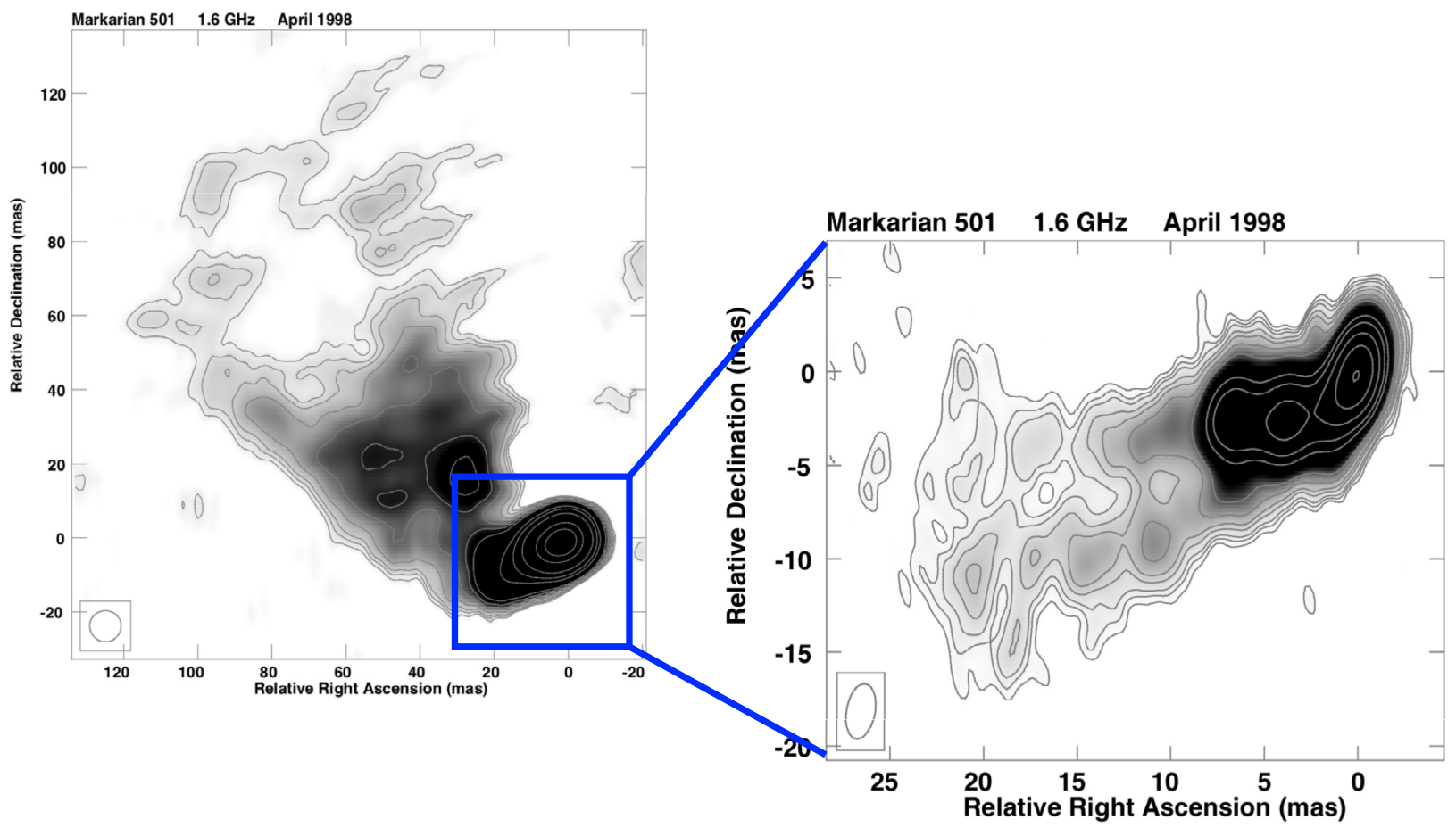

Figure 9. Limb brightening in Mrk501 before (right) and after (left) a large projected bend in the VLBI jet. The sizes of the Gaussian restoring beams are shown by the ellipses in the bottom left-hand corners of the images. Images are reprinted with permission from [62]. Copyright 2004 American Astronomical Society.

Limb brightening has been cited as evidence for a spine/sheath velocity difference (with the sheath emission beamed toward the observer) in a number of studies (e.g., [63] and references therein). At first glance, this seems a reasonable interpretation, given various theoretical studies and simulations taking into account mass loading at the jet edges and interactions with the ambient medium, which suggest that the jets are likely to be stratified [64-66]. However, a spine/sheath velocity difference is nevertheless a contrived way to explain limb brightening, for the following reason. Preferential Doppler beaming of the emission of a "sheath" around the jet toward the observer requires a special geometry, which could potentially come about in a few rare cases, but not as a general rule. The more limb-brightened AGN jets that are found, the less likely it is that this is the dominant explanation. An interesting example here is Mrk 501 (Figure 9), whose jet displays appreciable limb brightening both before and after a large projected bend in the jet. A changing jet direction implies changing Doppler factors for the "spine" and "sheath" of the jet: even if differential Doppler boosting were the explanation for the limb brightening, say, before the bend, the sheath emission should not also be preferentially Doppler boosted after the bend, since the viewing angle, and hence the Doppler factors for the "spine" and "sheath", have changed. This strongly suggests that the origin of limb brighening is instead related to some intrinsic property of a jet propagating through an ambient medium, such as a helical B field, mass loading or particle acceleration at the jet edges. Another relevant example here is $1 \mathrm{H} 0323+342$, in which Doi et al. [67] have detected limb brightening both before and after a local convergence of the jet which they identify with a recollimation shock; again, we would expect the relative Doppler factors for the "spine" and "sheath" to have changed as the jet plasma flows through this feature. 

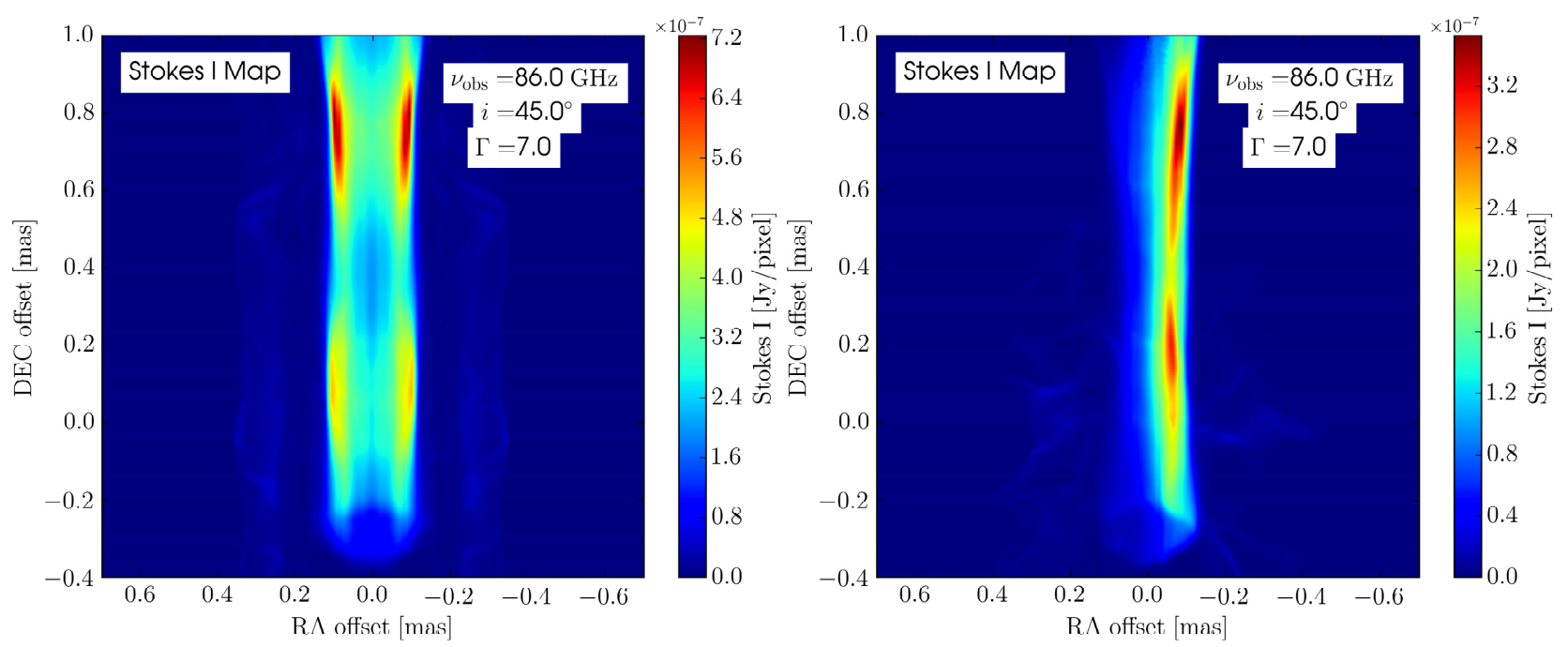

Figure 10. RMHD computations of limb brightening produced by the presence of a toroidal B-field component for the case of a purely toroidal field (left) and a helical field with an appreciable poloidal component (right), for a jet viewing angle of $45^{\circ}$. Images provided by Joanna Kramer.

Mrk 501 also provides an interesting case study for applying the principle of Occam's Razor. Parts of the jet display a spine-sheath polarization structure with an increase in the fractional polarization toward the jet edges, which could be due to a helical jet $\mathbf{B}$ field or shocks along the jet ridge line combined with shear at the jet edges. Mrk 501 also displays limb brightening, which could be due to a helical field, mass loading at the jet edges, or a velocity difference between a spine and sheath with the sheath beamed toward observer. We can already see that the presence of a helical jet $\mathbf{B}$ field is a common element in possible explanations for these two properties of the jet; the fact that a clear transverse RM gradient that encompasses both positive and negative RM values has been detected across the jet of Mrk 501 in multiple datasets [68,69] makes it likely that the inferred helical jet B field is also the origin of the observed polarization structure (see [41]), and of at least part of the observed limb brightening. Simulations are needed to determine how much of the limb brightening can be explained by a helical B field with specified parameters, and the possible role of other factors such as Kelvin-Helmholtz instability or mass loading.

\section{The Role of Circular Polarization in Determining the Full Three-Dimensional B-Field Structure}

The circular polarization of synchrotron radiation is intrinsically quite low. Magnetic fields of a few tenths of a Gauss, consistent with estimates of core B fields based on the frequency-dependent position of the VLBI core [70-73], and degrees of order of the fields of order $10 \%$, consistent with typical observed degrees of linear polarization, yield degrees of circular polarization $(\mathrm{CP}) m_{c}$ of no more than a few tenths of a percent. In fact, a substantial minority (approximately 15\%) of radio-loud AGNs display low, but significant levels of $\mathrm{CP}$, typically a few tenths of a percent [74]. While some of the observed CP could, in principle, be explained as intrinsic CP from synchrotron radiation, some of the observed $m_{c}$ values are too high to be explained in this way, requiring $\mathbf{B}$ fields that are implausibly high or implausibly well ordered; $m_{c}$ values in excess of $1 \%$ have been observed in some cases [74-76]. Thus, another mechanism must be operating in at least some cases. It is believed that this mechanism is Faraday conversion of linear to circular polarization in a magnetized plasma [77]. Faraday conversion operates when a linearly polarized electromagnetic wave passes through magnetized plasma. In fact, under similar conditions, Faraday conversion is considerably more efficient at generating CP than the synchrotron mechanism, and this fact alone implies that essentially all the observed parsec-scale CP in AGN cores and jets is generated via Faraday conversion. 
Faraday conversion is analogous to Faraday rotation. However, while Faraday rotation comes about due to the different rates of propogation of the RCP and LCP components of the incident polarized wave and depends on the ambient B-field component along the line of sight, Faraday conversion comes about due to the different rates of propogation of the components of the wave $\mathbf{E}$ field parallel and perpendicular to the ambient magnetic field, and depends on the ambient $\mathbf{B}$ field transverse to the line of sight [78]. The angle between the plane of linear polarization of the incident radiation $(\mathbf{E})$ and the ambient $\mathbf{B}$ field determines the sign of the CP produced. This angle must differ from 0 or $90^{\circ}$ in order to have conversion operate-otherwise no delay is introduced between the two synchrotron E-field components. This can place constraints on B-field geometries that can efficiently give rise to $\mathrm{CP}$ via conversion.

It is interesting that Faraday conversion arises naturally in a helical jet $\mathbf{B}$ field configuration. Because the parts of a helical jet $\mathbf{B}$ field located at the far and near side of the jet relative to an observer have different directions, the $\mathbf{E}$ field for linearly polarized radiation emitted at the far side of the jet will lie at some angle to the $\mathbf{B}$ field at the near side of the jet, and so be partially converted to circularly polarized emission as it passes through the near side [78-81]. Thus, the detection of significant CP could be a sign of a helical jet $\mathbf{B}$ field.

Note that linear-polarization observations cannot yield full information about the direction of the associated synchrotron B field, since only the plane of polarization is measured. For example, the presence of a longitudinal field component can be inferred from observations, but not whether it is oriented inward or outward (or indeed both at once). This hinders inference of the direction of rotation of the central accretion disk from, e.g., observations of transverse RM gradients, which yield the direction of the toroidal field component: it is not possible to infer the direction of the rotation of the central black hole and accretion disk without knowing also whether the longitudinal field is inward or outward along the jet.

However, an interesting possibility arises when clear polarization structure, significant $\mathrm{CP}$ and transverse RM gradients are all detected in the same AGN. These properties can be analyzed together to uniquely determine the direction of the longitudinal component of the helical B field. Once this is known, this information can be used to deduce the direction of the rotation of the central black hole and its accretion disk. Gabuzda [82] carried out such an analysis for 12 AGNs, and found statistically equal numbers of outward and inward longitudinal B-field components, and statistically equal numbers of AGNs displaying inferred rotation directions clockwise and counter-clockwise projected onto the sky. At the same time, it was found that direction of the rotation is correlated with the direction of the longitudinal B field, with clockwise/counter-clockwise projected rotation being preferentially associated with inward/outward current. This produces a preferred orientation for the toroidal component of the resulting helical $\mathbf{B}$ field after the initial longitudinal B field is wound up, corresponding to inward current along the jet axis on parsec scales, as has been observed [25].

Gabuzda [82] found that a fairly high fraction of AGNs with detectable VLBI CP also have significant transverse RM gradients (25-30\%), supporting the idea that the CP and transverse RM gradients may have the same origin: a helical jet $\mathbf{B}$ field. A project is currently underway to apply the same type of analysis to a larger sample of AGNs displaying both significant CP and transverse RM gradients across their jets.

\section{Summary}

AGN jets are expected theoretically to carry helical B fields, which come about due to the winding up of an initial longitudinal field component by the rotation of the central black hole and accretion disk. There is now substantial observational evidence that many, possibly all, AGN jets carry helical B fields, primarily from the detection of statistically significant transverse Faraday rotation gradients across the jets of some 50 AGNs on parsec scales and about a dozen AGNs on larger scales of tens to thousands of parsec [19,25-27]. 
The direction of a transverse RM gradient across an AGN jet directly reflects the direction for the toroidal B field causing the gradient. The direction of the toroidal field, in turn, can be used to infer the direction of the net current flowing inside this toroidal field. The collected data on transverse RM gradients on parsec scales show that the current along the jet axis is almost always inward. At the same time, VLA and comparatively low-frequency VLBA observations show that outward currents predominate on decaparsec-kiloparsec scales in a more extended region surrounding the jet. This is consistent with a threedimensional magnetic-field structure with one region of helical $\mathbf{B}$ field lying inside another, with the directions of the toroidal B-field components opposite in the inner and outer regions of helical field. Gabuzda et al. [25] have noted that this arrangement of fields and current is similar to that of a co-axial cable. The directions of the transverse RM gradients across the two kiloparsec-scale jets of 5C 4.114 and the existence of a preferred direction for the jet currents support the idea that the initial B-field that is accreted has a dipolar character, as was also suggested by the simulations of McKinney and Blandford [42]. Further simulations of jet launching and propagation assuming the accretion of a dipolar-type $\mathbf{B}$ field, or the development of such a field through a mechanism such as the "cosmic battery" of Contopoulos et al. [18] and Christodoulou et al. [19], and allowing for the formation of a nested-helical-field structure would be very valuable.

Virtually all of the B-field structures observed in AGN jets on parsec scales can be explained by the presence of a helical jet $\mathbf{B}$ field component, with the longitudinal and toroidal components of this helical field giving rise to longitudinal and transverse $\mathbf{B}$-field structures projected onto the sky. At the same time, some observed polarization structures could also be explained by the action of local factors, such as shocks, shear with the medium through which the jet propagaes, and jet bending. It can be challenging to identify the origin of specific observed polarization structures with certainty. The most promising approach is to also take into consideration the distributions of the degree of polarization and the spectral index, the jet morphology, the Faraday rotation distribution around the jet, and the possible presence of transverse RM gradients (a toroidal B-field component) across the jet.

Prominent limb brightening has been detected in a number of relatively nearby AGNs when observed with sufficiently high angular resolution. Although this has often been interpreted as reflecting different velocities for the central "spine" of the jet and a "sheath" around the jet, with the sheath emission Doppler boosted toward the observer, this explanation is contrived. It is more natural to suppose that the origin of the limb brightening is some inherent property of the jets themselves, such as their helical B fields or mass loading at the jet edges, which may be accompanied by the development of Kelvin-Helmholtz instability, magnetic reconnection and particle acceleration.

It is virtually certain that the small but significant amounts of circular polarization observed in the cores and inner jets of AGNs are produced by Faraday conversion of a small fraction of the linear polarization into circular polarization. This mechanism is much more efficient at producing circular polarization than the synchrotron mechanism, under equivalent conditions. The operation of Faraday conversion requires different orientations for the $\mathbf{B}$ fields in the source and conversion regions, and a helical jet $\mathbf{B}$ field is one example of a field geometry that ensures this and can thus facilitate this process. Joint analyses of the distribution of linear polarization, circular-polarization sign and the direction of transverse RM gradients can provide a powerful tool for reconstructing the full three-dimensional structures of helical B fields carried by AGN jets, and in correctly inferring the direction of rotation of the central black hole.

Funding: No funding was received to support this work.

Acknowledgments: I would like to thank both referees for their thorough and helpful reviews of this paper.

Conflicts of Interest: The authors declares no conflict of interest. 


\section{References}

1. Kellermann, K.I.; Sramek, R.; Schmidt, M.; Shaffer, D.B.; Green R. VLA observations of objects in the Palomar Bright Quasar Survey. Astron. J 1989, 98, 1195-1207. [CrossRef]

2. Lister, M.L.; Homan, D.C. MOJAVE: Monitoring of Jets in Active Galactic Nuclei with VLBA Experiments. I. First-Epoch 15 GHz Linear Polarization Images. Astron. J 2005, 130, 1389-1417. [CrossRef]

3. Pacholczyk, A.G. Radio Astrophysics; W. H. Freeman: San Franciso, CA, USA, 1970.

4. Wardle, J.F.C. The Variable Rotation Measure Distribution in 3C 273 on Parsec Scales. Galaxies 2018, 6, 5. [CrossRef]

5. Blandford R.D.; Königl, A. Relativistic jets as compact radio sources. Astrophys. J. 1979, 232, 34-48. [CrossRef]

6. Janssen, M.; Falcke, H.; Kadler, M.; Ros, E.; Wielgus, M.; Akiyama, K.; Baloković, M.; Blackburn, L.; Bouman, K.L.; Chael, A.; et al. Event Horizon Telescope observations of the jet launching and collimation zone in Centaurus A. Nat. Astron. 2021, in press. [CrossRef]

7. The Event Horizon Telescope Collaboration. First M87 Event Horizon Telescope Results. I. The Shadow of the Supermassive Black Hole. Astrophys. J. Lett. 2019, 875, L1-L17. [CrossRef]

8. Lister, M.L.; Homan, D.C.; Hovatta, T.; Kellermann, K.I.; Kiehlmann, S.; Kovalev, Y.Y.; Max-Boerbeck, W.; Pushkarev, A.B.; Readhead, A.C.S.; Ros, E.; et al. Mojave XVII. Jet kinematics and parent population properies of relativistically beamed radio-loud blazars. Astrophys. J. 2019, 874, 43-61. [CrossRef]

9. Blandford, R.D.; Payne, D.G. Hydromagnetic flows from accretion disks and the production of radio jets. Mon. Not. R. Astron. Soc. 1982, 199, 883-903. [CrossRef]

10. Blandford, R.D.; Znajek, R.L. Electromagnetic extraction of energy from Kerr black holes. Mon. Not. R. Astron. Soc. 1977, 179, 433-456. [CrossRef]

11. Nakamura, M.; Uchida, Y.; Hirose, S. Production of wiggled structure of AGN radio jets in the sweeping magnetic twist mechanism. New Astron. 2001, 6, 61-78. [CrossRef]

12. Lovelace, R.V.E.; Li, H.; Koldoba, A.V.; Ustyugova, G.V.; Romanova, M.M. Poynting Jets from Accretion Disks. Astrophys. J. 2002, 572, 445-455. [CrossRef]

13. Tchekhovskoy, A.; Bromberg, O. Three-dimensional relativistic MHD simulations of active galactic nuclei jets: Magnetic kink instability and Fanaroff-Riley dichotomy. Mon. Not. R. Astron. Soc. 2016, 461, L46-L50. [CrossRef]

14. Barniol Duran, R.; Tchekhovskoy, A.; Giannios, D. Simulations of AGN jets: Magnetic kink instability versus conical shocks. Mon. Not. R. Astron. Soc. 2017, 469, 4957-4978. [CrossRef]

15. Zamaninasab, M.; Clausen-Brown, E.; Savolainen, T.; Tchekhovskoy, A. Dynamically important magnetic fields near accreting supermassive black holes. Nature 2014, 510, 126-128. [CrossRef]

16. Tchekhovskoy, A.; Narayan, R.; McKinney, J.C. Efficient generation of jets from magnetically arrested accretion on a rapidly spinning black hole. Mon. Not. R. Astron. Soc. 2011, 418, L79-L83. [CrossRef]

17. McKinney, J.C.; Tchekhovskoy, A.; Blandford, R.D. General relativistic magnetohydrodynamic simulations of magnetically choked accretion flows around black holes. Mon. Not. R. Astron. Soc. 2012, 423, 3083-3117. [CrossRef]

18. Contopoulos, I.; Christodoulou, D.; Kazanas, D.; Gabuzda, D.C. The Invariant Twist of Magnetic Fields in the Relativistic Jets of Active Galactic Nuclei. Astrophys. J. 2009, 702, L148-L152. [CrossRef]

19. Christodoulou, D.; Gabuzda, D.; Knuettel, S.; Contopoulos, I.; Kazanas, D.; Coughlan, C. Dominance of outflowing electric currents on decaparsec to kiloparsec scales in extragalactic jets. Astron. Astrophys. 2016, 591, 61-71. [CrossRef]

20. Marscher, A.P.; Jorstad, S.G. Frequency and Time Dependence of Linear Polarization in Turbulent Jets of Blazars. Galaxies 2021, 9 , 27. [CrossRef]

21. O'Sullivan, S.P.; Brown, S.; Robishaw, T.; Schnitzeler, D.H.F.M.; McClure-Griffiths, N.M.; Feain, I.J.; Taylor, A.R.; Gaensler, B.M.; Landecker, T.L.; Harvey-Smith, L.; et al. Complex Faraday depth structure of active galactic nuclei as revealed by broad-band radio polarimetry. Mon. Not. R. Astron. Soc. 2012, 421, 3300-3315. [CrossRef]

22. Perley, R.A.; Bridle, A.H.; Willis, A.G. High-resolution VLA observations of the radio jet in NGC 6251. Astrophys. J. Suppl. 1984, 54, 291-334. [CrossRef]

23. Blandford, R.D. Astrophysical Jets; Cambridge University Press: Cambridge, UK, 1993; p. 26.

24. Asada, K.; Inoue, M.; Uchida, Y.; Kameno, S.; Fujisawa, K.; Iguchi, S.; Mutoh, M. A Helical Magnetic Field in the Jet of 3C 273. Publ. Astron. Soc. Jpn. 2002, 54, L39-L43. [CrossRef]

25. Gabuzda, D.C.; Nagle, M.; Roche, N. The jets of AGN as giant coaxial cables. Astron. Astrophys. 2018, 612, 67-78. [CrossRef]

26. Knuettel, S.; Gabuzda, D.C.; O'Sullivan, S.P. Evidence for Toroidal B-Field Components in AGN Jets on Kiloparsec Scales. Galaxies 2017, 5, 61. [CrossRef]

27. Knuettel, S. Evolution of the Magnetic Field Structure in the Jet Outflows from Active Galaxies. Ph.D. Thesis, University College Cork, Cork, Ireland, 2020.

28. Taylor, G.B.; Zavala, R.T. Are There Rotation Measure Gradients Across Active Galactic Nuclei Jets? Astrophys. J. Lett. 2010, 722, L183-L187. [CrossRef]

29. Mahmud, M.; Coughlan, C.P.; Murphy, E.; Gabuzda, D.C.; Hallahan, D.R. Connecting magnetic towers with Faraday rotation gradients in active galactic nuclei jets. Mon. Not. R. Astron. Soc. 2013, 431, 695-709. [CrossRef]

30. Zavala, R.T.; Taylor, G.B. Time-Variable Faraday Rotation Measures of 3C 273 and 3C 279. Astrophys. J. 2001, 550, L147-L150. [CrossRef] 
31. Asada, K.; Inoue, M.; Kameno, S.; Nagai, H. Time Variation of the Rotation Measure Gradient in the 3C 273 Jet. Astrophys. J. 2008, 675, 79-82. [CrossRef]

32. Mahmud, M.; Gabuzda, D.C.; Bezrukovs, V. Surprising evolution of the parsec-scale Faraday Rotation gradients in the jet of the BL Lac object B1803+784. Mon. Not. R. Astron. Soc. 2009, 400, 2-12. [CrossRef]

33. Gómez, J.L.; Roca-Sogorb, M.; Agudo, I.; Marscher, A.P.; Jorstad, S.G. On the Source of Faraday Rotation in the Jet of the Radio Galaxy 3C 120. Astrophys. J. 2011, 733, 11-22. [CrossRef]

34. Hovatta, T.; Lister, M.L.; Aller, M.F.; Aller, H.D.; Homan, D.C.; Kovalev, Y.Y.; Pushkarev, A.B.; Savolainen, T. MOJAVE: Monitoring of Jets in Active Galactic Nuclei with VLBA Experiments. VIII. Faraday Rotation in Parsec-scale AGN Jets. Astron. J 2012, 144, 105-138. [CrossRef]

35. Lisakov, M.M.; Kravchenko, E.V.; Pushkarev, A.B.; Kovalev, Y.Y.; Savolainen, T.K.; Lister, M.L. An Oversized Magnetic Sheath Wrapping around the Parsec-scale Jet in 3C 273. Astrophys. J. 2021, 910, 35-42. [CrossRef]

36. Kravchenko, E.V. ; Kovalev, Y.Y.; Sokolovsky, K.V. Parsec-scale Faraday rotation and polarization of 20 active galactic nuclei jets. Mon. Not. R. Astron. Soc. 2017, 467, 83-101. [CrossRef]

37. Plavin, A.; Kovalev, Y.Y.; Kovalev, Y.A.; Troitsky, S. Observational Evidence for the Origin of High-energy Neutrinos in Parsec-scale Nuclei of Radio-bright Active Galaxies. Astrophys. J. 2020, 894, 101-113. [CrossRef]

38. Plavin, A.; Kovalev, Y.Y.; Kovalev, Y.A.; Troitsky, S. Directional Association of TeV to PeV Astrophysical Neutrinos with Radio Blazars. Astrophys. J. 2021, 908, 157-166. [CrossRef]

39. Hovatta, T.; Lindfors, E.; Kiehlmann, S.; Max-Moerbeck, W.; Hodges, M.; Liodakis, I.; Lähteemäki, A.; Pearson, T.J.; Readhead, A.C.S.; Reeves, R.A.; et al. Association of IceCube neutrinos with radio sources observed at Owens Valley and Metsähovi Radio Observatories. Astron. Astrophys. 2021, 650, 83-99. [CrossRef]

40. Gabuzda, D.C.; Roche, N.; Kirwan, A.; Knuettel, S.; Nagle, M.; Houston, C. Parsec scale Faraday-rotation structure across the jets of nine active galactic nuclei. Astron. Astrophys. 2017, 472, 1792-1801. [CrossRef]

41. Murphy, E.; Cawthorne, T.V.; Gabuzda, D.C. Analysing the transverse structure of the relativistic jets of active galactic nuclei. Mon. Not. R. Astron. Soc. 2013, 430, 1504-1515. [CrossRef]

42. McKinney, J.C.; Blandford, R.D. Stability of relativistic jets from rotating, accreting black holes via fully three-dimensional magnetohydrodynamic simulations. Mon. Not. R. Astron. Soc. 2009, 394, L126-L130. [CrossRef]

43. Gabuzda, D.C.; Knuettel, S.; Bonafede, A. Evidence for a toroidal magnetic-field component in 5C 4.114 on kiloparsec scales. Astron. Astrophys. 2015, 583, 96-100. [CrossRef]

44. Knuettel, S.; O'Sullivan, S.P.; Curiel, S.; Emonts, B.H.C. The magnetic field strength of the Faraday screen surrounding the radio galaxy Coma A. Mon. Not. R. Astron. Soc. 2019, 482, 4606-4616. [CrossRef]

45. Lyutikov, M.; Pariev, V.I.; Gabuzda, D.C. Polarization and structure of relativistic parsec-scale AGN jets. Mon. Not. R. Astron. Soc. 2005, 360, 869-891. [CrossRef]

46. Gabuzda, D.C. Parsec-Scale Jets in Active Galactic Nuclei. In The Formation and Disruption of Black Hole Jets; Astrophysics and Space Science Library; Springer: Cham, Switzerland, 2015; Volume 414, pp. 117-142.

47. Pushkarev, A.B.; Gabuzda, D.C.; Vetukhnovskaya, Y.N.; Yakimov, V.E. Spine-sheath polarization structures in four active galactic nuclei jets. Mon. Not. R. Astron. Soc. 2005, 356, 859-871. [CrossRef]

48. Gabuzda, D.C.; Pushkarev, A.B.; Cawthorne, T.V. Analysis of $\lambda=6 \mathrm{~cm}$ VLBI polarization observations of a complete sample of northern BL Lacertae objects. Mon. Not. R. Astron. Soc. 2000, 319, 1109-1124. [CrossRef]

49. Healy, F. Multi-Epoch 18-22 cm VLBA Observations of Several BL Lac Objects. Master's Thesis, University College Cork, Cork, Ireland, 2013.

50. Laing, R. A model for the magnetic-field structure in extended radio sources. Mon. Not. R. Astron. Soc. 1980, 193, 439-449. [CrossRef]

51. Hughes, P.A.; Aller, H.D.; Aller, M.A. Polarized Radio Outbursts in Bl-Lacertae-Part Two-the Flux and Polarization of a PistonDriven Shock. Astrophys. J. 1985, 298, 301-315. [CrossRef]

52. Hughes, P.A.; Aller, H.D.; Aller, M.A. Synchrotron Emission from Shocked Relativistic Jets. II. A Model for the Centimeter Wave Band Quiescent and Burst Emission from BL Lacertae. Astrophys. J. 1989, 341, 68-79. [CrossRef]

53. Marscher, A.P.; Gear, W.K. Models for high-frequency radio outbursts in extragalactic sources, with application to the early 1983 millimeter-to-infrared flare of 3C 273. Astrophys. J. 1985, 298, 114-127. [CrossRef]

54. Laing, R.A.; Bridle, A.H. Systematic properties of decelerating relativistic jets in low-luminosity radio galaxies. Mon. Not. R. Astron. Soc. 2014, 437, 3405-3441. [CrossRef]

55. Clausen-Brown, E.; Lyutikov, M.; Kharb, P. Signatures of large-scale magnetic fields in active galactic nuclei jets: Transverse asymmetries. Mon. Not. R. Astron. Soc. 2011, 415, 2081-2092. [CrossRef]

56. Attridge, J.M.; Roberts, D.H.; Wardle, J.F.C. Radio Jet-Ambient Medium Interactions on Parsec Scales in the Blazar $1055+018$. Astrophys. J. Lett. 1999, 518, L87-L90. [CrossRef]

57. Sironi, L.; Rowan, M.E.; Narayan, R. Reconnection-driven Particle Acceleration in Relativistic Shear Flows. Astrophys. J. Lett. 2021, 907, L44-L49. [CrossRef]

58. Kramer, J.A.; MacDonald, N.R. Ray-Tracing in Relativistic Jet Simulations: A Polarimetric Study of Magnetic Field Morphology and Electron Scaling Relations. Astron. Astrophys. 2021, submitted. 
59. Takahashi, K.; Toma, K.; Kino, M.; Nakamura, M.; Hada, K. Fast-spinning Black Holes Inferred from Symmetrically Limbbrightened Radio Jets. Astrophys. J. 2018, 868, 82-101. [CrossRef]

60. Walker, R.C.; Hardee, P.E.; Davies, F.B.; Ly, C.; Junor, W. The Structure and Dynamics of the Subparsec Jet in M87 Based on 50 VLBA Observations over 17 Years at 43 GHz. Astrophys. J. 2018, 855, 128-163. [CrossRef]

61. Giovannini, G.; Savolainen, T.; Orienti, M.; Nakamura, M.; Nagai, H.; Kino, M.; Giroletti, M.; Hada, K.; Bruni, G.; Kovalev, Y.Y.; et al. A wide and collimated radio jet in 3C84 on the scale of a few hundred gravitational radii. Nat. Astron. 2018, 2, 472-477. [CrossRef]

62. Giroletti, M.; Giovannini, G.; Feretti, L.; Cotton, W.D.; Edwards, P.G.; Lara, L.; Marscher, A.P.; Mattox, J.R.; Piner, B.G.; Venturi, T. Parsec-Scale Properties of Markarian 501. Astrophys. J. 2004, 600, 127-140. [CrossRef]

63. Giroletti, M.; Giovannini, G.; Cotton, W.D.; Taylor, G.B.; P/'erez-Torres, M.A.; Chiaberge, M.; Edwards, P.G. The jet of Markarian 501 from millions of Schwarzschild radii down to a few hundreds. Astron. Astrophys. 2008, 488, 905-914. [CrossRef]

64. Nakamura, M.; Asada, K.; Hada, K.; Pu, H.-Y.; Noble, S.; Tseng, C.; Toma, K.; Kino, M.; Nagai, H.; Takahashi, K.; et al. Parabolic Jets from the Spinning Black Hole in M87. Astrophys. J. 2018, 868, 146-173. [CrossRef]

65. Chatterjee, K.; Liska, M.; Tchekhovskoy, A.; Markoff, S.B. Accelerating AGN jets to parsec scales using general relativistic MHD simulations. Mon. Not. R. Astron. Soc. 2019, 490, 2200-2218. [CrossRef]

66. Huang, L.; Pan, Z.; Yu, C. Toward a Full MHD Jet Model of Spinning Black Holes. II. Kinematics and Application to the M87 Jet. Astrophys. J. 2020, 894, 45-56. [CrossRef]

67. Doi, A.; Hada, K.; Kino, M.; Wajima, K.; Nakahara, S. A Recollimation Shock in a Stationary Jet Feature with Limb-brightening in the Gamma-Ray-emitting Narrow-line Seyfert 1 Galaxy 1H 0323+342. Astrophys. J. Lett. 2018, 857, L6-L11. [CrossRef]

68. Gabuzda, D.C.; Murray, É.; Cronin, P.J. Helical magnetic fields associated with the relativistic jets of four BL Lac objects. Astrophys. J. 2004, 351, L89-L93. [CrossRef]

69. Croke, S.M.; O'Sullivan, S.P.; Gabuzda, D.C. The parsec-scale distributions of intensity, linear polarization and Faraday rotation in the core and jet of Mrk501 at 8.4-1.6 GHz. Mon. Not. R. Astron. Soc. 2010, 402, 259-270. [CrossRef]

70. Kovalev, Y.Y.; Lobanov, A.P.; Pushkarev, A.B.; Zensus, J.A. Opacity in compact extragalactic radio sources and its effect on astrophysical and astrometric studies. Astron. Astrophys. 2008, 483, 759-768. [CrossRef]

71. O'Sullivan, S.P.; Gabuzda, D.C. Magnetic field strength and spectral distribution of six parsec-scale active galactic nuclei jets. Mon. Not. R. Astron. Soc. 2009, 400, 26-42. [CrossRef]

72. Sokolovsky, K.V.; Kovalev, Y.Y.; Pushkarev, A.B.; Lobanov, A.P. A VLBA survey of the core shift effect in AGN jets. I. Evidence of dominating synchrotron opacity. Astron. Astrophys. 2011, 532, 38-68. [CrossRef]

73. Pushkarev, A.B.; Hovatta, T.; Kovalev, Y.Y.; Lister, M.L.; Lobanov, A.P.; Savolainen, T.; Zensus, J.A. MOJAVE: Monitoring of Jets in Active galactic nuclei with VLBA Experiments. IX. Nuclear opacity. Astron. Astrophys. 2012, 545, 113-127. [CrossRef]

74. Homan, D.C.; Lister, M.L. MOJAVE: Monitoring of Jets in Active Galactic Nuclei with VLBA Experiments. II. First-Epoch 15 GHz Circular Polarization Results. Astron. J 2006, 131, 1262-1279. [CrossRef]

75. Homan, D.C.; Wardle, J.F.C. High Levels of Circularly Polarized Emission from the Radio Jet in NGC 1275 (3C 84). Astrophys. J. 2004, 602, L13-L16. [CrossRef]

76. Vitrishchak, V.M.; Gabuzda, D.C.; Algaba, J.C.; Rastorgueva, E.A.; O'Sullivan, S.P.; O’Dowd, A. The 15-43 GHz parsec-scale circular polarization of 41 active galactic nuclei. Mon. Not. R. Astron. Soc. 2008, 391, 124-135. [CrossRef]

77. Jones, T.W.; O'Dell, S.L. Transfer of polarized radiation in self-absorbed synchrotron sources. I. Results for a homogeneous source. Astrophys. J. 1977, 214, 522-539. [CrossRef]

78. Gabuzda, D.C.; Vitrishchak, V.M.; Mahmud, M.; O'Sullivan, S.P. Radio circular polarization produced in helical magnetic fields in eight active galactic nuclei. Mon. Not. R. Astron. Soc. 2008, 384, 1003-1014. [CrossRef]

79. Wardle, J.F.C.; Homan, D.A. The nature of jets: Evidence from circular polarization observations. In Particles and Fields in Radio Galaxies; Astron. Soc. Pac.: San Francisco, CA, USA, 2001; pp. 152-163.

80. Ensslin, T.A. Does circular polarisation reveal the rotation of quasar engines? Astron. Astrophys. 2003, 401, 499-504. [CrossRef]

81. Homan, D.C. Inverse Depolarization: A Potential Probe of Internal Faraday Rotation and Helical Magnetic Fields in Extragalactic Radio Jets. Astrophys. J. 2012, 757, L24-L28. [CrossRef]

82. Gabuzda, D.C. Determining the Jet Poloidal B Field and Black-Hole Rotation Directions in AGNs. Galaxies 2018, 6, 9-14. [CrossRef] 